\title{
Increasing Efficiency of Recruitment in Early Parkinson's Disease Trials: A Case Study Examination of the STEADY-PD III Trial
}

\author{
Sarah Berk ${ }^{\mathrm{a}}$, Brittany L. Greco ${ }^{\mathrm{b}}$, Kevin Biglan ${ }^{\mathrm{c}}$, Catherine M. Kopil ${ }^{\mathrm{a}}$, Robert G. Holloway ${ }^{\mathrm{b}}$, \\ Claire Meunier ${ }^{\mathrm{d}}$ and Tanya Simuni ${ }^{\mathrm{e}}$ * \\ ${ }^{a}$ The Michael J. Fox Foundation, New York, NY, USA \\ ${ }^{\mathrm{b}}$ Center for Human Experimental Therapeutics, University of Rochester, Rochester, NY, USA \\ ${ }^{\mathrm{c}}$ Eli Lilly and Company, Indianapolis, IN, USA \\ ${ }^{\mathrm{d}}$ Evidation Health, San Francisco, CA, USA \\ ${ }^{\mathrm{e}}$ Department of Neurology, Northwestern University, Feinberg School of Medicine, Chicago, IL, USA
}

Accepted 27 September 2017

\begin{abstract}
.
Background: Challenges in clinical trial recruitment threaten the successful development of improved therapies. This is particularly true in Parkinson's disease (PD) studies of disease modification where the population of interest is difficult to find and study design is more complex.

Objective: This paper seeks to understand how STEADY PD III, a National Institute of Neurological Disorders and Stroke (NINDS) funded phase 3 trial evaluating the efficacy of isradipine as a disease modifying agent for PD, was able to recruit their full target population 6 months ahead of schedule.

Methods: STEADY PD III aimed to enroll 336 individuals with early stage idiopathic PD within 18 months using 57 sites across the United States and Canada. The study included a 10\% NIH minority recruitment goal. Eligible participants agreed to be followed for up to 36 months, complete 12 in-person visits and 4 telephone visits. A Recruitment Committee of key stakeholders was critical in the development of a comprehensive recruitment strategy involving: multi-modal outreach, protocol modifications and comprehensive site selection and activation. Efforts to increase site-specific minority recruitment strategies were encouraged through additional funding.

Results: A total of 336 individuals, including 34 minorities, were enrolled within 12 months -6 months ahead of the projected timeline. Quantitative analysis of recruitment activity questionnaires found that of the sites that completed them $(n=54)$, (20.4\%) met goals, $(24.1 \%)$ exceeded goals, and $(55.6 \%)$ fell below projected goals. Referral sources completed at time of screening indicate top four study referral sources as: site personnel (53.8\%); neurologists (24\%); Fox Trial Finder (10.2\%); and communications from The Michael J. Fox Foundation (3.9\%).

Conclusions: STEADY PD III serves as an important example of methods that can be used to increase clinical trial recruitment. This research highlights a continued need to improve site infrastructure and dedicate more resources to increased participation of minorities in clinical research.
\end{abstract}

Keywords: Recruitment methods, Parkinson's disease, disease modifying trials

\footnotetext{
*Correspondence to: Tanya Simuni, MD, Professor of Neurology, Parkinson's Disease and Movement Disorders Center, Department of Neurology, Northwestern University, Feinberg
}

School of Medicine, 710 North Lake Shore Drive, 1126, Chicago, IL 60611, USA. Tel.: +1 312503 2970; Fax: +1 312908 5073;

E-mail: Tatyana.Simuni@nm.org. 


\section{INTRODUCTION}

Development of effective strategies to slow progression of Parkinson's disease (PD) is an area of major unmet need. Clinical trials of disease modifying therapies are one of the most active areas for clinical research in PD, with an average of 2 studies per year, over the past 25 years [1]. Unfortunately, no studies to date have yielded positive, conclusive results. A still nascent understanding of the pathogenesis and heterogeneity of the disease combined with a lack of biomarkers and suboptimal animal models contribute to lack of success. Other challenges include slow recruitment into complex and often lengthy clinical trials. This is further compounded by the desire to enroll individuals who are newly diagnosed with PD, often before they have initiated symptomatic treatment, as most clinical trialists believe that this stage of the disease is the best therapeutic window for slowing or halting disease progression. In light of these challenges, this paper will examine methods that enabled full recruitment in STEADY PD III, a National Institute of Neurological Disorders and Stroke (NINDS)-funded Phase 3 study of isradipine in PD.

\section{MATERIALS AND METHODS}

\section{Study organization}

STEADY-PD III is an ongoing 36-month doubleblind randomized, placebo-controlled study of isradipine in participants with early PD at baseline not receiving or requiring symptomatic therapy. The projected time period for enrollment was 18 months. Details of the scientific rationale and study design have been recently published [2]. The study is being conducted at 57 Parkinson Study Group (PSG) sites in North America (including Canada). The PSG is an independent consortium of scientific investigators committed to the cooperative planning, implementation, analysis and reporting of controlled clinical trials and other research in PD [3]. Northwestern University serves as the Clinical Coordination Center (Simuni, Principal Investigator) and University of Rochester Clinical Trials Coordination Center (CTCC) as the Data Coordination Center (DCC) (Holloway, Principal Investigator) and Biostatistical Core (BCC) (Oakes, Principal Investigator). The Data and Safety Monitoring Board (DSMB), an independent group of experts appointed by the NIH, perform oversight and monitoring of trial data and participant safety. Recruitment efforts were overseen by a Recruitment Committee comprised of: 1) STEADY PD III Principal Investigators; 2) STEADY PD III project managers from the CTCC; 3) site representatives (investigators and/or coordinators); 4) representatives from The Michael J. Fox Foundation (MJFF); 5) representatives from NINDS; and 6) patient advocates.

\section{Study participants}

STEADY PD III aimed to enroll 336 individuals with early stage idiopathic PD above the age of 30 at the time of diagnosis, male or female, having been diagnosed for a period of less than 3 years, not currently receiving symptomatic therapy (levodopa, dopamine agonist or MAO-B inhibitors), and not projected to require PD symptomatic therapy (ST) for at least 3 months from baseline visit. To participate in the study, eligible participants agreed to be followed for a period of up to 36 months and complete 12 inperson visits and 4 telephone visits. The projected recruitment period was based on experience with the previously completed studies that targeted a similar PD population.

\section{Recruitment materials}

To facilitate outreach and awareness efforts, the STEADY PD III Recruitment Committee developed a suite of materials packaged as a "Recruitment Toolkit." The toolkit was composed of patient engagement and clinician engagement materials - two groups identified as key target audiences for outreach and awareness efforts. Patient engagement materials included the following: 1) Appointment cards; 2) Patient Education Frequently Asked Questions (FAQs); 3) Site Brochures; 4) Site Posters; 5) Patient geared Slide Deck; 6) Patient Talking Points; 7) Site Press Release; and 8) Thank You Cards. The clinician targeted materials included the following: 1) Primary Care Provider (PCP) Notification Letter; 2) Physician Outreach Letter; and 3) Physician geared Slide Deck. More information on the description and implementation of these materials can be found in (Table 1). The content developed for these materials was intended to: educate and increase target audience awareness of STEADY PD III and how/where they could find more information; address potential key points or concerns of the target audience(s) around clinical trial participation; and appeal to the 
Table 1

Recruitment toolkit

\begin{tabular}{ll}
\hline Asset Name & Addresses \\
\hline Patient Facing & Main points \\
Appointment Cards & Knowledge gaps \\
Patient Education FAQ & Knowledge gaps and motivations \\
Site Brochure & Knowledge gaps and motivations \\
Site Poster & Main Points, Knowledge Gaps and Motivations \\
Patient Facing Slide Deck & Main Points, Knowledge Gaps and Motivations \\
Patient Talking Points & Knowledge Gaps and Motivations \\
Site Press Release & Main Points and Motivations \\
Thank You Cards & \\
Clinician Facing & Knowledge Gaps \\
PCP Notification Letter & Main Points, Knowledge Gaps, and Motivations \\
Physician Outreach Letter & Main Points, Knowledge Gaps, and Motivations \\
MD Facing Slide Deck &
\end{tabular}

motivations of the target audience(s) for participating in or referring to STEADY PD III.

\section{Design features and procedures to facilitate recruitment}

Throughout the clinical trial planning phase, the STEADY PD III Steering Committee (SC), in conjunction with the Recruitment Committee, implemented the following methods to facilitate and increase recruitment, including: 1) critical review of the complexity of the research protocol; 2) rigorous selection and activation of the clinical trial sites; and 3) development of a comprehensive outreach and awareness strategy. These methods are further explored below.

\section{Reduced protocol complexity}

Cognizant of the negative impact of an overly complex protocol design $[4,5]$, STEADY PD III SC worked to reduce the stringency of eligibility criteria and lessen participant burden while maintaining a careful balance between investigative and scientific rigor. These objectives were accomplished through the following protocol modifications: 1) reducing the length of time before projected initiation of symptomatic therapy (ST) from 6 months to 3 months; 2) extending the duration of participant follow up to 36 months; and 3) using change in total UPDRS score in the medications' ON state, if ST had been initiated, as the primary outcome measure. These changes differentiate the STEADY PD III protocol from other trials of PD disease modification which tended to be 12-24 months in duration and used either time to initiation of ST or last UPDRS prior to initiation of ST as outcome measures [1]. The STEADY PD III design allowed study participants to initiate ST while still participating in their randomized treatment assignment, and allowed for continuation in the study, even if the study drug had to be discontinued for any reason. In addition to increased efficiency in recruitment, these modifications made study results generalizable to a larger PD patient population. Modifications to the STEADY PD III protocol occurred prior to site activation, and were the result of an iterative review process between the SC and the Data and Safety Monitoring board (DSMB). The decision to reduce the time before projected initiation of ST was made following concerns by the DSMB that ST was being commenced at lower thresholds (among physicians and patients) and that this criterion might serve as a barrier to enrollment.

\section{Rigorous site selection and activation}

To facilitate site selection, STEADY PD III SC developed a list of qualifications considered necessary for successful recruitment into the study. These qualifications included: 1) available PD population within the clinic; 2) expected monthly enrollment and total enrollment goals; 3) confirmed prior experience enrolling newly diagnosed PD patients; 4) recruitment metrics from recent participation in PD studies; and 5) experience of site staff. These qualifications contributed to study SC decision to recruit sites from within the Parkinson's Study Group (PSG). A study participation interest form was circulated, and out of the 132 credentialed PSG sites, 87 applied. Applications were rank ordered based on the above listed criteria, and a total of 60 sites (55 active and 5 back up) were selected by the Steering Committee. Following the selection process, a recruitment activity questionnaire (Table 2) was circulated to participating sites to assess: strengths, weaknesses, and areas for additional financial and non-financial support as it 
Table 2

Recruitment activity questionnaire

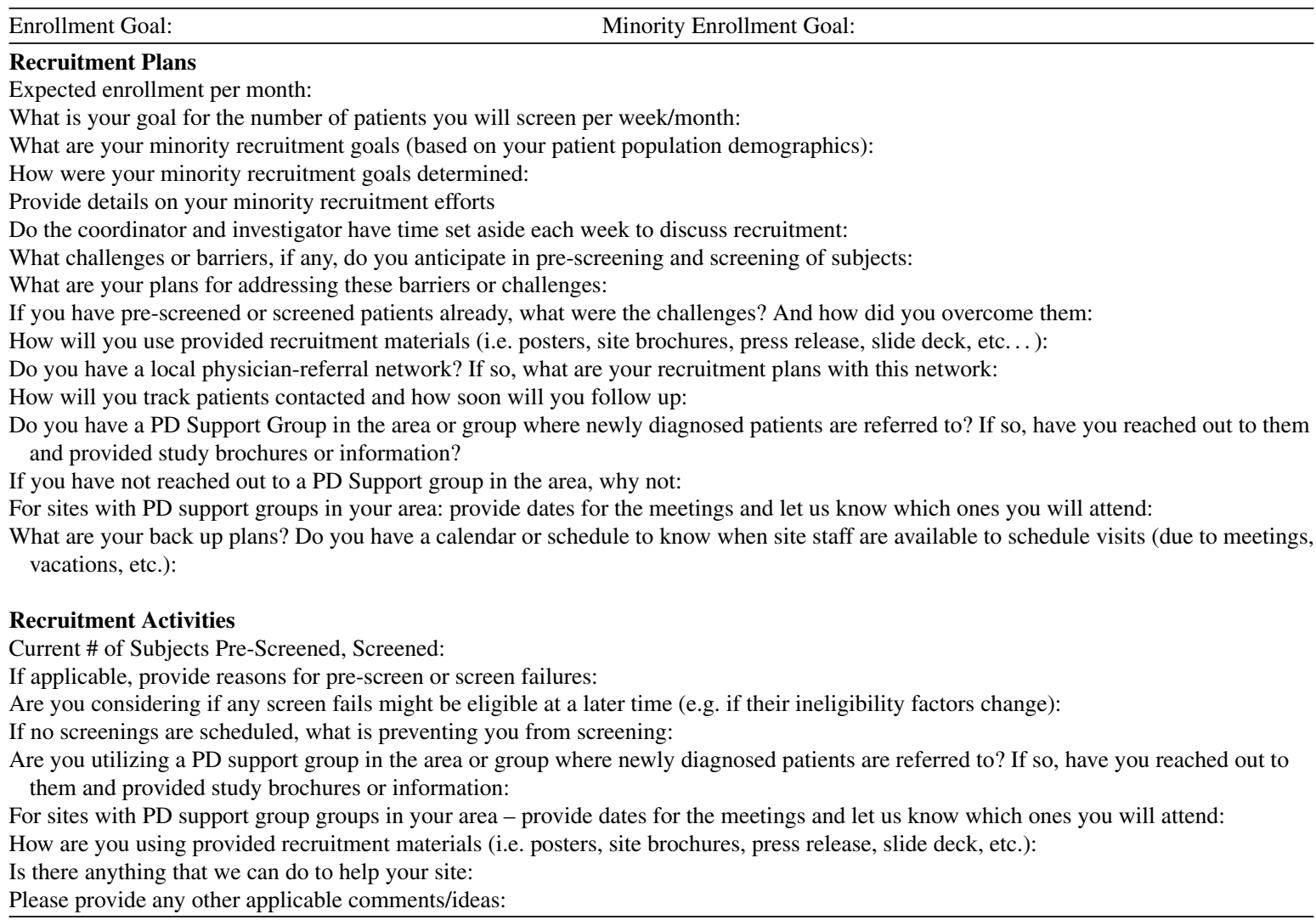

pertained to recruitment. This information was used to develop site-specific recruitment plans, and materials for the Recruitment Toolkit. All recruitment materials were submitted to the Institutional Review Board (IRB) at the University of Rochester (home of the CTCC) along with the study protocol and informed consent making them ready for sites' IRB submissions early in the regulatory process. Once IRB approved, the study management team printed out, laminated, and mailed a complete Recruitment Toolkit to each of the clinical trial sites, along with other study materials and regulatory forms. Printing and distribution of the Recruitment Toolkit aimed to mitigate the site-level administrative burden of developing and producing recruitment materials. A study initiation meeting took place September 2014 where sites received an in-depth overview of strategies for recruitment and minority recruitment goals, training on the research protocol, background information on the study drug, and logistics for monitoring and communication. After receiving IRB approval, sites participated in individual start-up calls with the CTCC team to review recruitment strategies and available tools. An enrollment goal of four participants per site was set at initiation, but was changed to competitive enrollment shortly thereafter. After activation, sites were required to participate in monthly coordinator calls where they reviewed and modified recruitment strategies, determined new or additional outreach opportunities; discussed ways to leverage technology for recruitment; assessed why participants did not consent; and facilitated the development or revision of recruitment materials.

\section{Comprehensive outreach and awareness strategy}

Early on in the planning process, the STEADY PD III team identified and engaged key stakeholders from across the recruitment landscape to provide input on constituent motivations, knowledge gaps and outreach methods. This group became the Recruitment Committee and received consultation from the National Parkinson Foundation (NPF) and the Parkinson Disease Foundation (PDF). The committee developed a multi-modal recruitment strategy 
aimed at educating individuals in the PD community about STEADY PD III and increasing awareness of resources related to the trial. This strategy was implemented through: 1) in person meetings and events with community groups, physician networks and support groups; and 2) development of a heightened online presence using mixed media outlets. Recruitment Toolkit materials such as the "Health Care Provider Outreach Letter" and the "Patient geared Slide Deck" facilitated outreach efforts, as did grassroots peer engagement via MJFF's Fox Trial Finder (FTF) Ambassadors, PDF's Parkinson's Advocates in Research (PAIR), and the Muhammad Ali Foundation's community leaders. A greater online presence was cultivated through: 1) creation of a study specific website, STEADYPD3.com (https://steadypd3.com/); 2) press releases (templates were provided in the Recruitment Toolkit) posted to websites such as NINDS; 3) use of Fox Trial Finder - MJFF's online trial matching service that enables volunteers to connect with trial teams (https://foxtrialfinder.michaeljfox.org/); and 4) webinars and podcasts hosted by the STEADY PD III study PIs and broadcast to MJFF and NPF networks.

\section{Minority recruitment}

At all phases of study launch and execution, emphasis was placed on strategies to maximize minority recruitment - an historical challenge in PD trials. As a part of site selection study leadership carefully evaluated minority recruitment statistics from previously completed NINDS funded PD de novo studies, as well as demographic data provided by sites. Those sites that had experienced success at enrolling minority participants and had access to a higher percentage of minority volunteers were selected for study implementation. On a local level, sites were encouraged to collaborate with minority community leaders, translate materials into French and Spanish, and to contact local lay and/or professional organizations serving minority populations to schedule lunch or dinner events where study information was shared. In addition to these outreach efforts, study leadership created a "Minority Recruitment Application" that sites could apply to for additional funding to support local minority outreach. As part of the application process sites were encouraged to submit proposals describing the minority recruitment event or strategy that they were planning to undertake as well as the cost for the event, which could fall into the following funding ranges: $\$ 500-1000$, $\$ 1000-\$ 1500,>\$ 1500$.

\section{Clinical trial sites and factors impacting qualitative analysis of recruitment performance}

Goals for anticipated monthly and total enrollment were collected through the recruitment activity questionnaires, and a comparison of projected enrollment numbers vs. actual enrollment numbers was completed post recruitment. This comparison was used to determine whether sites met, exceeded or fell below their enrollment goal(s). Once grouped, qualitative analysis of site responses across enrollment categories was performed to see if any major themes emerged.

\section{RESULTS}

A study enrollment report (Fig. 1) shows a steeper slope of actual vs. anticipated enrollment, reflective of a recruitment period accelerated by 6 months. In addition, the prespecified goal of $10 \%$ minority recruitment was met.

Recruitment success in STEADY-PD III was bolstered by a multitude of subject referral sources as reflected in (Fig. 2). Referral sources were recorded at the time of screening and logged into case report forms. These data indicate the top four referral sources as: Site personnel (53.8\%); Neurologists (24\%); FTF (10.2\%); and MJFF Communications $(3.9 \%)$.

Analysis of MJFF communications that took place prior to and throughout the recruitment period provides insight into the role of mixed media in generating awareness of the trial, and directing individuals to resources for learning more about participation. Starting in March of 2014, MJFF, with study leadership, released a podcast that reported the compound Isradipine was moving to Phase III testing, and recruitment would begin later that same year. The podcast was downloaded by a total of $(2,043)$ iTunes listeners and followed by an uptick in STEADYPD3.com site traffic beginning in May 2014 and peaking in July 2014 (Fig. 3). One of the steepest peaks occurred in January 2015 following a December 2014 webinar by MJFF that focused on therapies with the potential to slow or stop Parkinson's progression and highlighted the STEADY PD III trial. A third peak took place November 2015 following an MJFF webinar and podcast on studies to slow/stop Parkinson's disease on October 2015. While proprietary ownership of web analytics makes it a challenge to compare web traffic of STEADY PD III to that of 


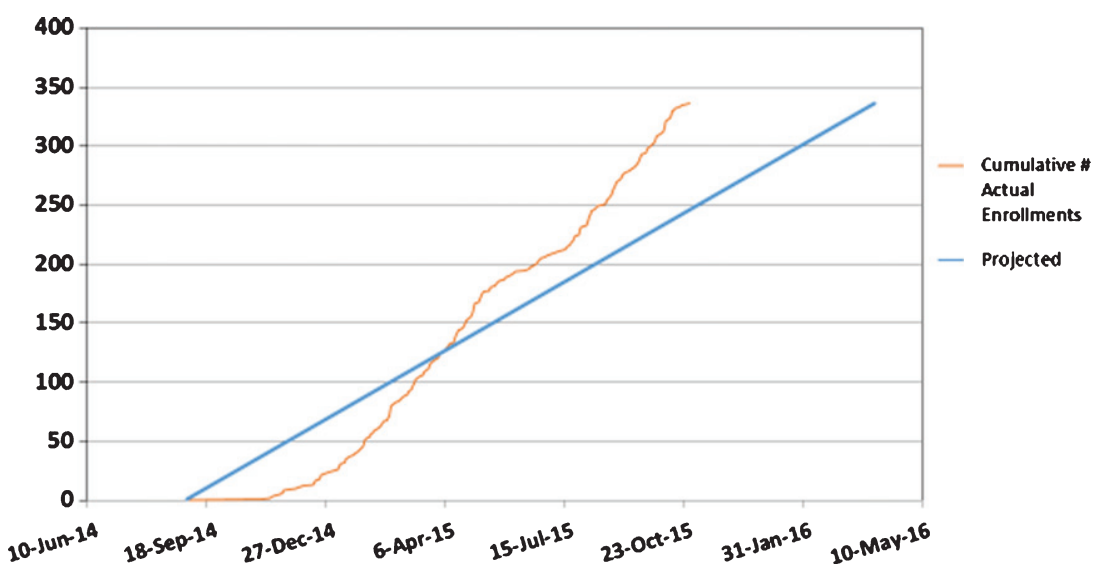

Fig. 1. Actual vs. Projected Enrollment.

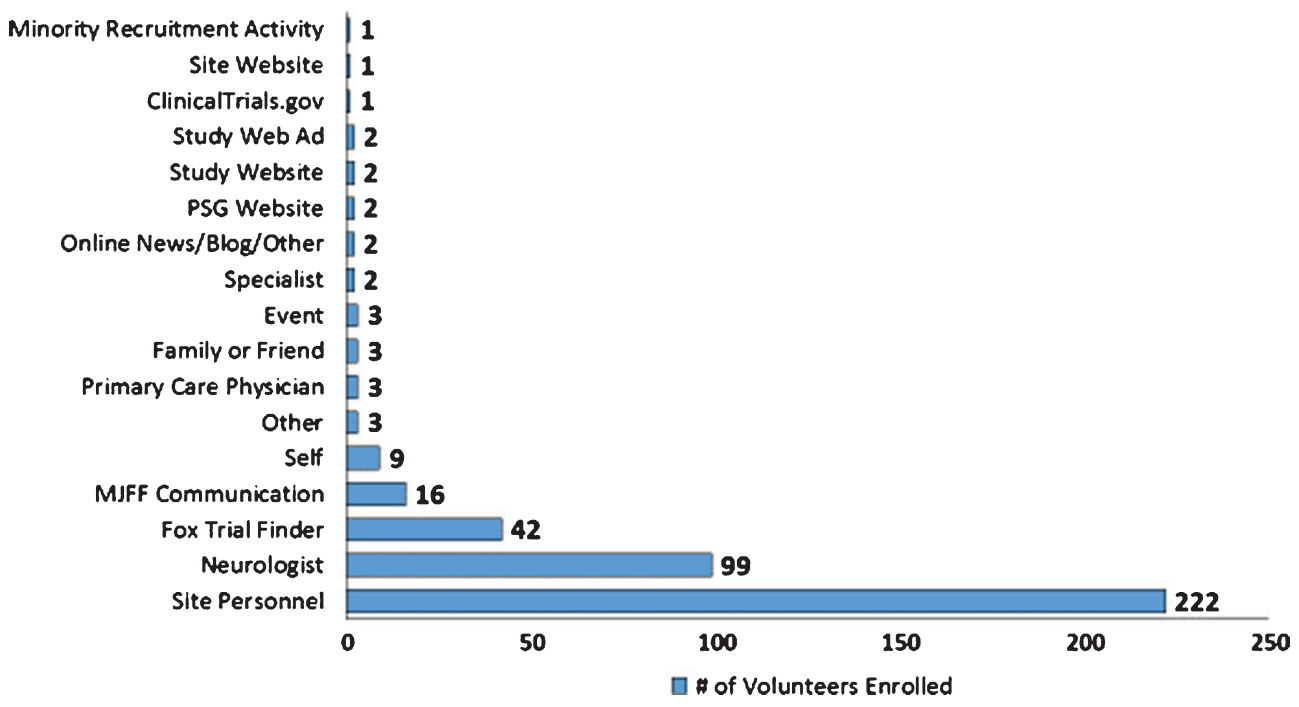

Fig. 2. Referral Sources: Enrolled STEADY PD III Volunteers.

other studies, a better understanding of visitor traffic to and behaviour on clinical trial websites would help to elucidate their utility as a recruitment tool and remains an important area for future research.

Comparison of actual enrollment vs projected enrollment for those sites that filled out recruitment activity questionnaires $(n=54)$ revealed that $(20.4 \%)$ met their goals, $(24.1 \%)$ exceeded their goals, and (55.6\%) fell below projected goals. Major themes from the qualitative analysis were as follows: 1) goal setting; 2) site team communication; 3) anticipated recruitment barriers; 4) use of recruitment toolkit materials; 5) community engagement; and 6) patient tracking methods. Our analysis revealed that a consistent challenge, across sites, was the ability to accurately predict recruitment goals. That only
$20 \%$ of sites met their recruitment goal compared to the $80 \%$ that exceeded or under-enrolled further supports this finding. Communication was similar across sites that exceeded enrollment and under-enrolled, with both groups citing weekly check-ins between the investigator and the coordinator. All sites, independent of recruitment results, reported similarly on anticipated barriers, which included: concerns around finding individuals that met the eligibility criteria (i.e. de novo, not already taking medication and/or early PD), and issues with logistics, such as transportation, scheduling, workload levels, and finding time to contact patients. Sites that exceeded or under-enrolled most frequently reported no potential barriers, which may have contributed to or mitigated successful achievement of enrollment goals. The recruitment 


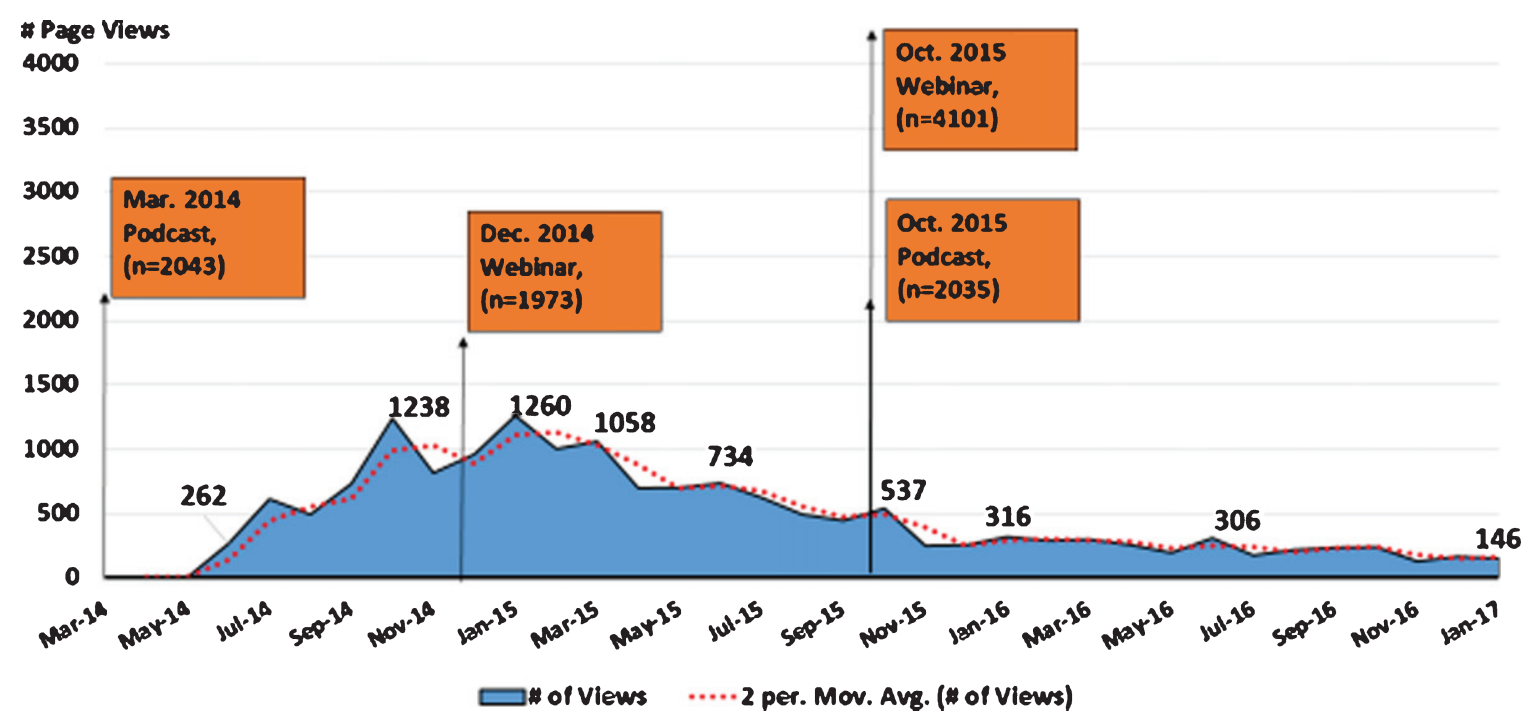

Fig. 3. STEADYPD3.Com and MJFF Mixed Media Outreach.

strategy for all three enrollment groups involved a multi-modal approach, with particular emphasis on outreach to clinician groups and placement of materials in areas of high volunteer traffic (primarily at the site or in clinics). All enrollment groups included an element of social media (list servs and websites) and participation in local events, although the latter two elements were not emphasized as much as the former. In regards to community engagement, connections with patient support groups were cited more frequently than with physician referral networks. Finally, all enrollment groups reported some form of tracking method for conducting outreach to and screening of volunteers, with the majority of sites reporting use of a paper log and or excel spreadsheet. Only one or two sites in the exceeded enrollment and under enrollment groups reported use of a secure database.

\section{DISCUSSION}

STEADY PD III SC was able to successfully recruit full target population six months ahead of schedule due to a pre-specified comprehensive recruitment plan and involvement of key stakeholders early in the planning phase of the clinical trial. These actions allowed SC to identify study and site level barriers that had the potential to negatively impact recruitment, and develop a strategy to mitigate them. Key components of this strategy consisted of: 1) a reduction in protocol complexity; 2) implementation of a comprehensive outreach and awareness campaign; and 3) rigorous selection and activation of clinical trial sites.

The fact that the protocol complexity was identified as one of the study level barriers is not surprising. Clinical trial complexity is an issue that has been on the rise over the past 20 years. Research by the Tuft's Center for the Study of Drug Development found that in this time, the average number of inclusion criteria have increased threefold and the number of unique procedures per protocol have increased by $6.5 \%$ [4]. This growing level of complexity has negatively impacted recruitment, resulting in a 53\% increase in the times between study initiation and completion, and a $16 \%$ reduction in enrollment rates [4]. The authors posit that this trend is attributable to a shift in focus to chronic diseases, where clinical endpoints are difficult to measure and the target population for recruitment is highly specific. Additional factors, such as study design lend themselves to more complex protocols, with studies of efficacy tending towards greater stringency in eligibility criteria to allow them to recruit for a more homogenous patient population and facilitate assessment of the outcome of interest [6]. STEADY PD III, based on the study objectives, was inherently more complex seeking to demonstrate efficacy in a disease state that currently lacks biomarkers and among a patient population that can be challenging to recruit (i.e. those recently diagnosed and not yet on ST). Modifications to the study protocol addressed this barrier by loosening the stringency of the inclusion and exclusion 
criteria without jeopardizing scientific integrity. This in turn, increased the number of individuals who were eligible to participate in the study, lowered the burden for potential participants, and broadened the pool of individuals from which sites could recruit. The positive impact of these modifications on recruitment can be seen in a comparison of the low rate of screen failures from STEADY PD III (18.6\%) to that of parallel studies of disease modification in patients with early Alzheimer's disease, which can reach rates upwards of $(85 \%)$ [7].

Gaps in patient knowledge around the availability of clinical research and the benefits of research participation were also identified as a barrier to recruitment. A 2001 survey by Harris Interactive found $85 \%$ of patients were unaware that participation in a clinical trial was an option at the time of diagnosis, and $75 \%$ of these patients would have been willing to enroll had they known it was a possibility [8]. Central to addressing this barrier was involvement of key stakeholder groups who leveraged their unique capabilities to engage with communities and raise awareness. Stakeholders such as PDF maximized peer to peer engagement via the PAIR program and local events such as the Brain and Health Fair and the Unity Walk; NPF harnessed the power of social media through webinars and press releases; MJFF leveraged technology such as Fox Trial Finder to connect volunteers to trial teams; and The Muhammad Ali Foundation focused on increased participation of historically underrepresented minority populations via community engagement, translation of materials, and outreach via the "Southwestern Parkinson's Newsletter." The use of localized, grassroots events, and social media activities, combined with a proactive approach to recruitment helped to engage and make aware a broader swath of the population than would have been possible for clinical trial sites alone. This approach also enabled study teams to connect with a more diverse patient population who obtain their information from a variety of media and news sources. The impact of these efforts on facilitating recruitment for the STEADY PD III trial are challenged by the data reflected in (Fig. 2) on self-reported referral sources. We posit that this is less about the efficacy of these efforts and more about the challenges stemming from memory recall bias in referral source attribution. Greater efforts such as interviewer training, better referral source definition, and alternative means of data collection should be considered for future recruitment campaigns to improve the accuracy of attribution [9].
A comparison of STEADY PD III enrollment figures to 2013 Tufts Center for the Study of Drug Development (CSDD) benchmarks demonstrated that the number of STEADY PD III sites that exceeded enrollment goals $(24.1 \%)$ were nearly double that of the CSDD benchmark (13\%), while the percentage of STEADY PD III sites that met their goals $(20.4 \%)$ was nearly half that of the $(39 \%)$ CSDD benchmark [10]. The STEADY PD III sites that under-enrolled $(55.6 \%)$ were $\sim 19$ percentage points above the CSDD average (37\%) [10]. These data suggest that: 1) there were a number of high performing sites, approximately one quarter of those participating in the study, that played a large role in meeting recruitment goals; 2) study leadership accurately identified site level characteristics that were needed for successful recruitment and targeted sites that exhibited those characteristics; and 3) site activation processes, such as customized recruitment plans, helped to address the majority of site-level barriers, but some still remain. These data also indicate that sites may experience challenges accurately predicting their recruitment goals, and it may be of benefit for study leadership to consider making recruitment competitive from trial initiation to avoid any inefficiencies or additional costs [11]. Two additional areas for site level enhancements are: greater automation of tools and organizational systems to track recruitment efforts, which would allow sites to more quickly respond to high or low volumes of study participants; and increased engagement with clinician groups to foster long term referral networks.

While the STEADY PD III approach to clinical trial recruitment was a success, a few key limitations should be taken into consideration. A reduction in protocol complexity may not be scientifically feasible for all clinical research studies. If considering, the impact that the modifications will have on scientific rigor and validity should be weighed before attempting. STEADY PD III also had a relatively low level of participant burden, which could make these results somewhat less applicable to studies requiring more complex procedures. Another limitation, especially when trying to quantify the impact of partnerships on recruitment numbers is that referral sources may suffer from memory recall bias; multiple sources could have led to an individual's awareness and participation in a study. Consistent and descriptive categorical definitions should be provided to site staff to better assist individuals in determining the source that led to their study involvement. Finally, the drug being tested for STEADY PD III had been repurposed, meaning it 
had been FDA approved for other indications which may have positively skewed attitudes and perceptions of safety for participants.

\section{CONCLUSION}

STEADY PD III serves as an important example of methods that can be used to increase clinical trial recruitment. Involvement of key stakeholders early on is important for learning about constituent motivations and barriers to participation. There is growing interest in improving the value and efficiency of the clinical trial process, including the identification of site qualities (geography, investigator characteristics) and the multi-modal methods (e.g., recruitment tools, social media platforms) needed to optimize recruitment success and improve clinical trial efficiency [12]. Development of a comprehensive recruitment strategy can be used to build awareness and bridge knowledge gaps; and technology, both new platforms and social media, has an ever-growing role in the recruitment landscape. Additional research is needed to better understand the impact of multi-modal outreach efforts (e.g. channels that are most effective for recruitment efforts and how they compare to one another; messaging that resonates with volunteers and why), and how these lessons might be applied to future recruitment campaigns. Gaps remain on ways to improve site infrastructure and methods to enhance recruitment, and on-going efforts should be dedicated to increase participation of minorities in clinical research.

\section{CONFLICT OF INTEREST}

The authors have no conflict of interest to report.

\section{REFERENCES}

[1] AIDakheel A, Kalia LV, \& Lang AE (2014) Pathogenesistargeted, disease-modifying therapies in Parkinson disease. Neurotherapeutics, 11, 17.

[2] Biglan KM, Oakes D, Lang AE, Hauser RA, Hodgeman K, Greco B, Lowell J, Rockhill R, Shoulson I, Venuto C, Young D, Simuni T, \& Parkinson Study Group STEADYPD III Investigators (2017) A novel design of a Phase III trial of isradipine in early Parkinson disease (STEADY-PD III). Ann Clin Transl Neurol, 4, 8.

[3] Parkinson Study Group. What is the Parkinson Study Group (PSG), Flight 9 Creative, http://www.parkinson-study-gro up.org/about, Accessed October 2017.

[4] Getz K (2008) Protocol Design Trends and their Effect on Clinical Trial Performance. RAJ Pharma.

[5] Getz K (2014) Improving protocol design feasibility to drive drug development economics and performance. Int J Environ Res Public Health, 11, 11.

[6] Singal AG, Higgins PD, \& Waljee AK (2014) A primer on effectiveness and efficacy trials. Clin Transl Gastroenterol, 5 , e45.

[7] Babic T, \& Riordan HJ (2016) Improving Screen Failure and Recruitment Rate in Alzheimer's Disease Clinical Trials. VertMarkets, Inc.

[8] National Institutes of Health. The Need for Awareness of Clinical Research, U.S. Department of Health and Human Services. Available from: https://www.nih.gov/health-infor mation/nih-clinical-research-trials-you/need-awareness-cli nical-research. Accessed October 2, 2017.

[9] Hassan E (2005) Recall bias can be a threat to retrospective and prospective research designs. Internet J Epidemiol, 3, number 2 .

[10] Tufts Center for the Study of Drug Development (2013) $89 \%$ of trials meet enrollment, but timelines slip, half of sites under-enroll. In Impact Report. CSDD Tufts University Online. http://csdd.tufts.edu/files/uploads/jan-feb_2013_ir_ summary.pdf

[11] Bienkowski RS, \& Goldfarb NM (2008) Screen failures in clinical trials: Financial roulette or the cost of doing business? J Clin Res Best Pract, 4, 1-4.

[12] Demaerschalk BM, Brown RD Jr, Roubin GS, Howard VJ, Cesko E, Barrett KM, Longbottom ME, Voeks JH, Chaturvedi S, Brott TG, Lal BK, Meschia JF, Howard G, \& CREST-2 Investigators (2017) Factors associated with time to site activation, randomization, and enrollment performance in a stroke prevention trial. Stroke, 48, 7. 